\title{
Design of the GPS Navigation Vehicle Terminal System
}

\author{
Min Peng ${ }^{1, a, *}$, Zhi Min Chen ${ }^{2, b}$ \\ ${ }^{1}$ School of Information Engineering, Wuhan Technology and Business University, Wuhan 430033, \\ PR China \\ ${ }^{2}$ College of Power Engineering, Naval University of Engineering, Wuhan 430033, PR China \\ a 21495396@qq.com, bczm12345678@yeah.net
}

Keywords: Car GPS navigation, GSM module, GPS module.

Abstract. The car's GPS navigation system is a part of intelligent transportation, it can satisfy the personalized requirements of users travel and effectively improve the problem of heavy traffic. In the face of huge car market, the potential of car navigation is immeasurable, and its development prospect is optimistic. This paper proposed a GPS navigation vehicle terminal system based on ST ARM and ST UPSD, and introduced in detail each part design of the GPS host, including the vehicles positioning communication module, power supply and drive systems of the peripheral equipment, infrared remote control module, display interface module, etc., and the design of the GPS vehicle handle module.

\section{Introduction}

At present, traffic congestion is a common problem, which the countries around the world met, simply increasing road infrastructure can not completely solve the traffic transportation condition, especially in the inner cities of more limited land resources. In order to solve the contradiction between vehicles and road, it is necessary to introduce modern electronic information technology to transportation systems, so the intelligent transportation System ITS arises at the historic moment. Application of GPS in ITS main reflected in vehicle navigation.

Car's GPS navigation system is a vehicular information platform for the integration of the set of satellite positioning system, wireless communication system and geographic information system, it through the received GPS information decoding to the vehicle's current position and motion, and then map matching with the electronic map stored in the equipment, results displayed on the LCD screen, so as to realize the location of the vehicle operation. The system consists of three parts that are automotive equipment, communication network, the command and dispatch center.

The focus of this paper is GPS vehicle terminal equipment, it can realize vehicle monitoring, anti-theft, alarm, navigation, display and get the current vehicle position (latitude and longitude), provide hands-free car phone and so on functions. 
This system is divided into GPS host (including the vehicles positioning communication module, power supply and drive systems of the peripheral equipment, infrared remote control module, display interface module), and GPS handle(mainly display interface module).

We can found that the GPS handle module and GPS mainframe module has some similar features, so the GPS handle is not a must. Be advised that papers in a technically unsuitable form will be returned for retyping. After returned the manuscript must be appropriately modified.

\section{Design of the GPS Host}

Host mainly completed the following work: through the received GPS information decoding to the vehicle's current location and movement state, and then map matching with the electronic map stored in the equipment, and the matching results displayed on the LCD screen, so as to realize the location of the vehicle operation. The device integrates module for wireless communication at the same time, through this module, vehicle not only can receive all kinds of instructions from scheduling command center, also can upload their own status information to the center, it provides a reliable means of communication for Real-time monitoring of vehicle scheduling command center. The hardware system consist eight parts including CPU, GSM module, GPS module, monitoring, FLASH, RS232 interface, the vehicle condition monitoring, burglar alarm and audio module. As shown in figure 1.

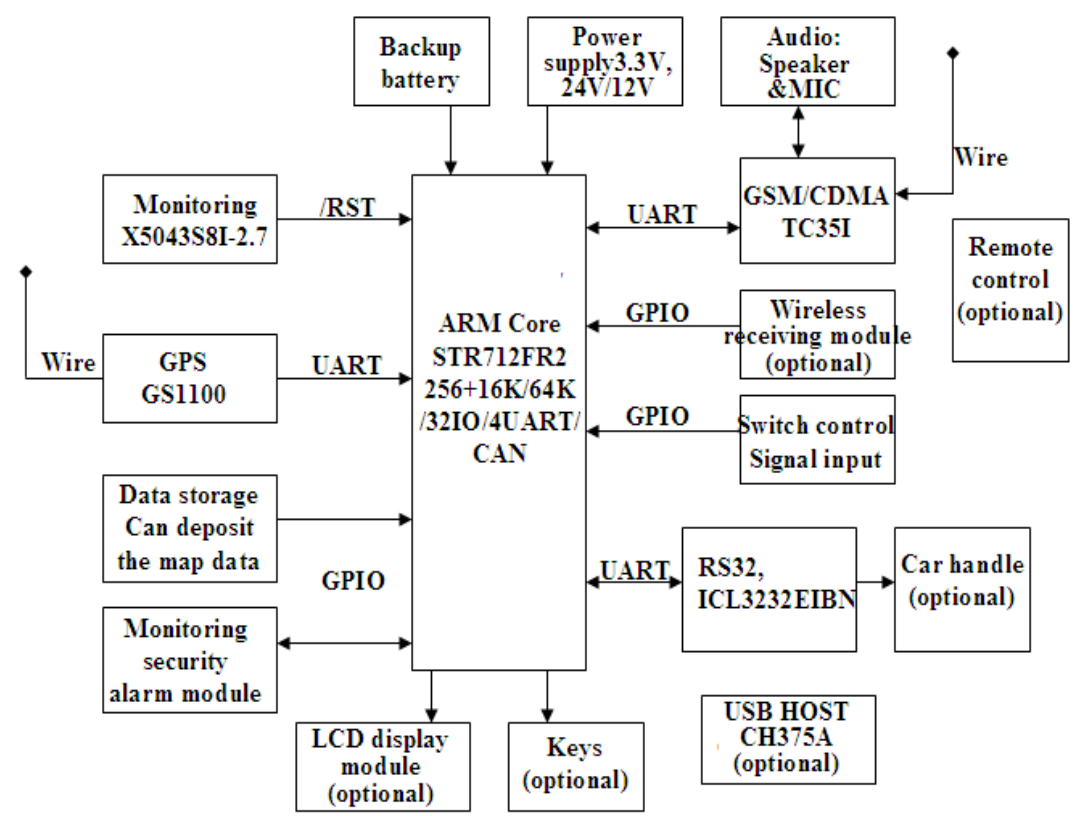

Figure 1 The host schematic diagram of car GPS navigation system.

\subsection{The $\mathrm{MCU}$}

CPU used STR712FR2 of ST (or STR710FZ2 with EMI to extend parallel storage module). STR712FR2 is launched low-priced 256k bytes flash version of ST with CAN interface - STR712, equipped with $16 \mathrm{k}$ bytes data flash memory and 64k bytes RAM. It's RAM/Flash balance was optimized of ST, and in the process of code development limitations to minimize the Flash applications, provides services to more complex applications. For the interface which don't need a USB or CAN, it can use STR715. Bare STR715 is a low-cost MCU, equipped with 64k bytes flash memory,16k bytes data flash memory and $16 \mathrm{k}$ bytes RAM. Based on ARM7TDMI 32 - bit RISC CPU industry standard STR71XF series is the ideal choice for embedded system, at the same time, 
it is also very suitable for the user interface, industrial automation systems, and POS machine, etc. STR71XF series provided up to $33 \mathrm{MHZ}$ directed flash and the sequence flash memory of $50 \mathrm{MHZ}$ Zero wait state, optimized the real-time application. In addition, STR712FR2 integrated rich peripherals, for example, a CAN2.0 controller, two I2C interface, two SPI interface, four UART, HDLC function, five timer, 12 bit ADC of 4 channel and 32 GPIO, some peripheral function to reduce the demand for peripheral devices in most applications. It can be used to store programs and character data, etc.

\subsection{GSM module}

At present, the domestic have begun to use the GSM module including Falcom A2D series, Wavecome WMO2 series, Siemens TC35 series, Ericsson DM10 / DM20 series, ZTE ZXGM18 series and so on, and the function of these modules and usage, witch difference is not big. Siemens TC35 module series has high performance, and has the domestic radio equipment examined. So this design chooses the TC35i Siemens TC35 series, functionally compatible with TC35, compact design, greatly reduce the volume of the user product. TC35i compatible with GSM $2 / 2+$, dualfrequency (GSM900 / GSM1800), RS232 data, accord with standard of ETSI GSM0707 and GSM0705, and easy to upgrade for the GPRS module. The module integrating radio frequency circuit and the base band, to provide customers with the standard of the AT command interface, for data, voice, short message and fax to provide fast, reliable and safe transmission, convenient user application development and design.

Between GSM module and microcontroller using a standard serial port to communicate with STR712FR2, high baud rate of communication can reach 115200 bit/s. Mainly through SIMCLK between GSM module and SIM card and SIMDATA signal lines for data communication. In order to guarantee the time interval is short as far as possible between send a short message and a short message to reach. it is best to choose the same telecom operators to provide SIM cards. When using GPRS function, still should choose the SIM card to support GPRS and open the GPRS service. GSM module also supports drive two microphone, two road speakers and a buzzer. One microphone and speakers can be connected to the handle of the receiver, in order to realize the function of car phone.

\subsection{GPS module}

In this paper, the GPS receiver of using is GS1100, the receiver has a set of serial input/output interface, serial input port is used to receive initialized instructions from the single chip microcomputer, after initialization is complete, the receiver will serially send GPS positioning information every two seconds through the outlet.GS1100 integrated antenna, and the interface is fairly simple with MCU, it can directly communicate with STR712FR2 serial port.

\subsection{Monitoring circuit}

Monitoring circuit X5043 can power on/off electric reset, with functions of programmable watchdog. $4 \mathrm{k}$ block Bit EEPROM used to store the vehicle initialization Settings.

\subsection{The map data storage module}

M25P64/32 stores electronic map data (including the color of the map information) downloaded from the Internet, according to the specific data format the ARM generates electronic 
maps and displays it on the LCD screen. It can also be used for the built-in black boxes GPS, storage driving records, extend the functionality of the GPS.

\subsection{2 interface}

Through a serial port to connect GPS host and GPS vehicle-mounted handle . interface IC choose ICL3232EIBN.

\subsection{System monitoring and anti-theft alarm module}

Through GPIO realize monitoring, anti-theft alarm. STR712FR2 GPIO can connect the guard against theft, air-conditions, alarm, circuit, circuit, pressure, temperature, central door lock, alarm monitoring control circuit of the vehicle. It can according to user requirements define the corresponding GPIO as a variety of sensors(such as vibration sensor) and switch quantity input/output, as well as the input/output of the alarm and braking system (such as warning horn output, extinguish fire relay output, vehicle anti-theft device interface, set/removal, open/close the door, backup relay output). Implementing an interface with vehicle anti-theft alarm, emergency alarm (SOS), medical service, Breakdown services, relay output, warning beep and ignition signal, Including the under-voltage detection alarm.

\subsection{Audio module}

GSM module supports to drive two microphone, two road speakers and a buzzer. One microphone and speakers can be connected to the handle of the receiver, in order to realize the function of car phone. It can also increase the audio op-amp and audio power amplifier module to improve output power, Such as MC4558 + LM4890 can achieve better audio effect.

\subsection{Power supply module}

The power supply of the Car GPS navigation system is generally directly from the car battery or igniter. Usually for $12 \mathrm{v}$ or $24 \mathrm{v}$ (there will be a great fluctuation in the process of start), general selection is DC/DC. , usually the main power supply in IC, choose $8 \mathrm{v}$ output, choose ON LM2576A.

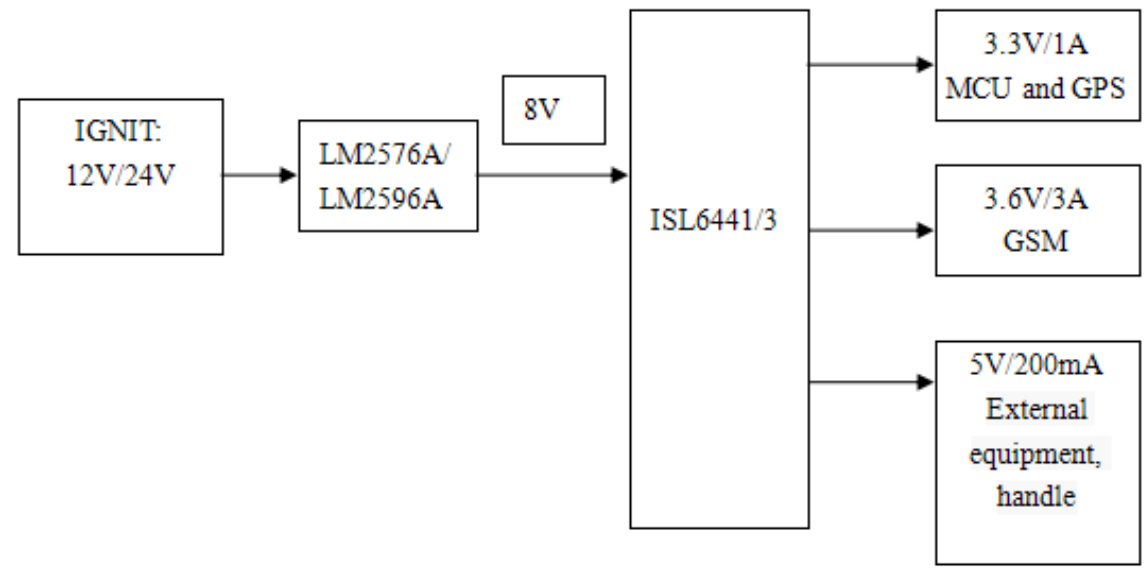

Figure 2 Power supply requirements and design of the GPS system.

GSM module power supply, considering the current GSM module launch of two ampere: 3.6 $\mathrm{X} 2=7 \mathrm{w}, 2$ a peak current, select LM2576A. Directly to the $12 \mathrm{~V} / 24 \mathrm{~V}$ to $3.6 \mathrm{~V}$. (due to different 
wireless module power demand is not the same as here you can choose the adjustable output IC, also can use multiple output, such as ISL4441/3)

MCU power supply: 3.3 X0.1 $=0.33 \mathrm{w}$. given certain allowance, can choose NCP500:150 ma / $3.3 \mathrm{~V}$.

Power supply scheme is shown in figure 2.

\section{The GPS Vehicle Handle}

Handle module can provide for the driver with a hands-free car phone and SMS transceiver, etc, it can receive speech signal and text messages from dispatching center, it can also transmit the driver and the car's voice signal and text messages, in this way can realize the voice communications and text messages of the vehicle and the center. When the vehicle was robbed, the driver can send robbery alarm information to the center, after receiving, the center can open the car's hands-free phones so as to realize To monitor the car's voice, Combined with character, it can realize Chinese short message function.

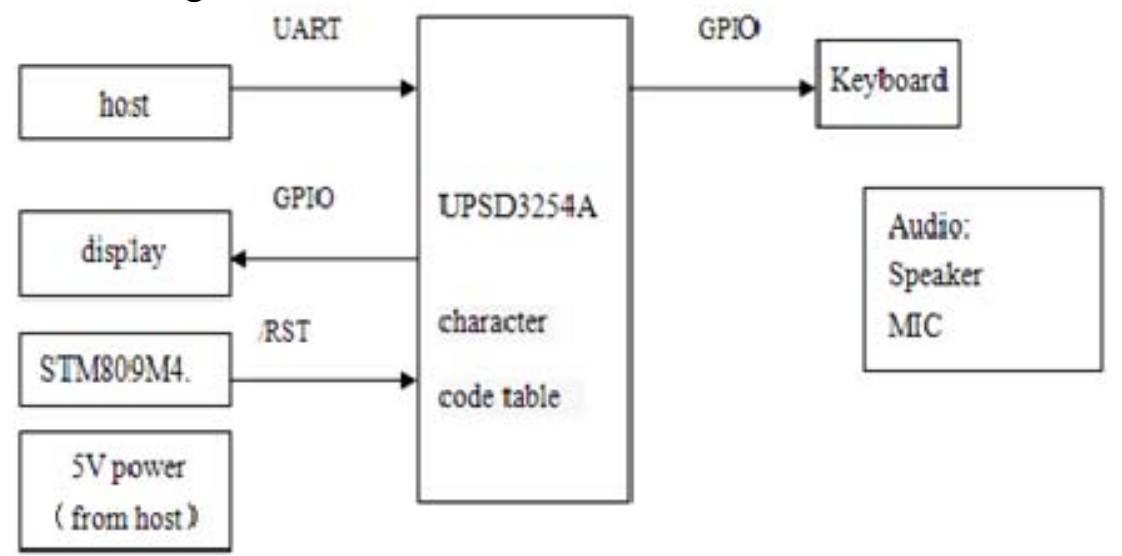

Figure 3 The handle module block diagram.

As figure 3, the CPU use UPSD3254A of ST, the chips compatible with MCU instructions of the INTEL51 series, can use C51 development tools. The frequency is $40 \mathrm{M}$, there were $256 \mathrm{k}+32$ $\mathrm{k}$ bytes FLASH program memory, the size of the SRAM is $32 \mathrm{k}$ byte on chip. Double RS232 serial interface, that is very easy to communicate and data calculate. Through serial port the UPSD directly communicate with host MCU of the GPS: STRF712FR, internal $288 \mathrm{k}$ Flash can be used to put a program and character (of course, can also use the lower part of the MCU and character of IC). Choose STM809M as the monitor IC, used for UPSD3254A on the working voltage of the electric reset and $5 \mathrm{v}$ power reset. Using ordinary black and white LCD display text messages. The keyboard used universal keyboard. GSM module supports drive two microphone, two road speakers and a buzzer. One microphone and speakers of the GSM can be connected to the handle of the receiver, in order to realize the function of car phone. $5 \mathrm{v}$ power supply can be obtained from GPS host directly.

\section{Acknowledgements}

At present, China's auto communications and navigation was still in the stage of market cultivation, smaller as a whole. Related facilities and resources is not perfect, Real-time traffic information releasing and providing perfect navigation electronic map with updating mechanism are lack of practical solutions and ways, thus the market promotion is difficult. But in the face of large car market, car navigation potential is immense, its development prospect is optimistic. 
According to the analysis of the present situation of domestic automobile GPS navigation market, market development trend, manufacturers in the aspects, the car's GPS navigation market will proceed to the next generation product planning, accelerate new product development progress, some have begun, and some new companies are also actively carry out work, hope to enter this industry. The rapid development of China's automobile industry will put the whole navigation system in automobile industry to a climax.

\section{References}

[1] Wang hua, Design of the BD2 / GPS dual-mode vehicle navigation terminal system, Digital technology application, 2013(3).

[2] Xia feng, Hu da, The design of the urban car GPS navigation system, computer and modernization,2004(3).

[3] Zhao jun, the design of the vehicle intelligent terminal of the Open-pit coal mine harvester, Electronics world, 2017.1.

[4] Teng wen, The optimal path search algorithm research of the vehicle navigation system. Automation and instrumentation, 2016.12.

[5] Yang lin guo, Design of a new type of car terminal positioning navigation system Based on GPS, BlueTooth, GPRS, Journal of anhui vocational \& technical college,2011.2. 